\title{
Cargo Aircraft Conceptual Design Optimisation Using a Flexible Computer-Based Scaling Approach
}

\author{
F. Schieck, D. Schmitt
}

\begin{abstract}
In the early design stages of a new aircraft, there is a strong need to broaden the knowledge base of the evolving aircraft project, allowing a profound analysis of the solution concepts and of the design driving requirements. The methodology presented in this paper provides a tool for increasing and improving in an exemplary manner the necessary information on cargo aircraft. By exchanging or adapting a few particular modules of the entire program system, the tool is applicable to a range scale of different aircraft types. In an extended requirement model, performance requirements are represented along with other operational requirements. An aircraft model is introduced in sufficient detail for conceptual design considerations. The computer-aided scaling methodology is explained, which, controlled by an optimisation module, automatically resizes the aircraft model until it optimally satisfies the requirements in terms of a selectable figure of merit. Typical results obtained at the end of the scaling are discussed together with knowledge gained during the process, and an example is given.
\end{abstract}

Keywords: aircraft conceptual design, aircraft scaling, mass growth factor, aircraft multidisciplinary design optimisation.

\section{Introduction}

The engineering design process of a new aircraft starts with a set of requirements which are to be met by one or several aircraft concepts that we may call solutions, being developed along the timeline. During the conceptual design phase, on which this paper is focused, various possible solutions are investigated, modified, abandoned or further developed. Simultaneously, several requirements may face a discussion, in which the required parameter values, and even the requirements themselves, are questioned and changed if necessary. Later, the preliminary design phase will follow, looking further into only a few particularly promising aircraft studies, the number of which will be reduced to a single concept to enter the detailed design phase. By then, the requirements will be far more rigid than during the conceptual design stage.

The design phases are characterised by steadily changing degrees of knowledge, freedom of design, and cost of change (Figure 1). Knowledge is information about the evolving aircraft project. Naturally, in the early design phase, it is incomplete or imprecise, so assumptions are often made. These assumptions, if incorrect, can lead to poor decisions that precipitate project failure, budget and/or schedule overruns, etc. Freedom of design is a measure of flexibility, or the degree to which changes in the aircraft characteristics are realistic. Cost of change refers to the resource allocation which is determined by the decision-making processes.

Key decisions made early in the design process determine a comparatively large number of aircraft parameters as well as a high percentage of the total cost committed, as [1] points out. Unfortunately, these decisions are often based on minimal knowledge and incomplete or inaccurate information. Necessary revisions in later design phases are significantly more expensive and complicated than are changes early in the process. So there is a desire to shift knowledge forward in the design timeline, enabling better substantiated decisions. This faster increase of design knowledge implies changes of improvement in the conceptual design stage, as these better substantiated decisions then meet a more flexible and less costly-to-change aircraft design state (as indicated by the arrows in Figure 1).

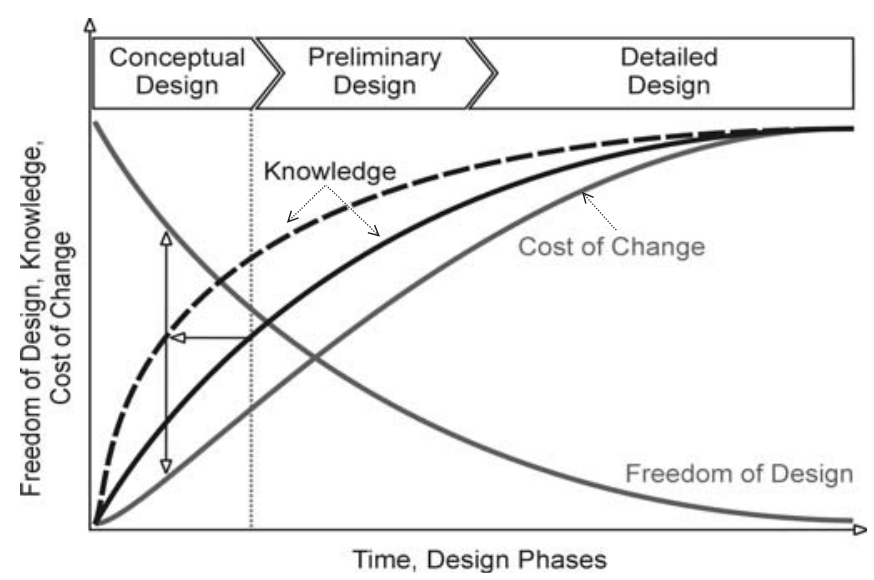

Fig. 1: Changes of chances during the conceptual design phase

In order to accomplish this, a tool is required to help increase and improve the information about the evolving aircraft project in the early design stages, allowing a sounder review of presented solutions and of the design driving requirements. One possible approach is an iterative scaling process, which will be described in detail below. Here, starting from a model reference aircraft not yet meeting all requirements, several parameters are resized - scaled - deliberately in each step, thus describing a scaled aircraft with new characteristics, which, in turn, are subject to investigation. This iteration is guided by the objective of an aircraft design which optimally satisfies the initial requirements with respect to a selectable figure of merit, e.g. total mass. At the same time, various design sensitivities become apparent along the iteration, adding to the desired information base.

At the Chair of Aeronautical Engineering of the Technische Universität München, the FASTR (lexible Aircraft Scaling To Requirements) program is currently being developed as a modern computer-aided approach to run this scaling process automatically, as will be described in detail 
in the following sections. In this context, aircraft requirements, which can be integrated into a requirement model, are introduced as design objectives in Section 2. Complementing the requirement model, an aircraft model is presented in a sufficient grade of detail for conceptual design considerations in Section 3. Subsequently, the FASTR core, an automated scaling algorithm, is described in Section 4. The results of this scaling process are discussed in Section 5, and an example is given in Section 6.

\section{Aircraft requirements as design objectives}

The engineering design process of a new aircraft begins with the specification, that has to be reached in the end with a certain technical solution. Hence, during all scaling efforts, the specification defines the design-guiding boundary conditions. A full specification consists of several requirements, most of them relating to performance. With few exceptions, e.g. a dedicated stealth aircraft, where stealthiness and low signatures may dominate the whole design [2], performance requirements can be considered strong design drivers in an aircraft project. The approach described herein will therefore set one focus on performance requirements, as will become apparent in Section 4.

These demanded performances can be divided basically into point performance requirements (Table 1) and mission performance requirements (Table 2). The former describe singular performance items which have to be satisfied at a single point in time with a fixed aircraft setup. The latter relate to performance requirements which have to be met in a mission context, along a flight profile, with e.g. a steadily changing fuel mass. For both requirement classes, various formula systems have been developed and have been published [3], [4].

Table 1: Point performance requirements

\begin{tabular}{|ll|}
\hline \multicolumn{2}{|c|}{$\begin{array}{c}\text { Point performance requirements } \\
\text { At any given fuel and payload percentage }\end{array}$} \\
\hline - Stall speed & - Sustainable load factor \\
- Take-off run length & - Specific excess power \\
- Turn rate & - Landing run length \\
\hline
\end{tabular}

Table 2: Mission performance requirements

$\left.\begin{array}{|l|l|}\hline \multicolumn{2}{|c|}{\text { Mission performance requirements }} \\ \hline \text { - Payload mass } & - \text { Climb rate } \\ \text { - Range } & - \text { Acceleration } \\ \text { - Cruise altitude } & - \text { Manoeuvres } \\ \text { - Cruise speed } & - \text { Store drop }\end{array}\right\} \begin{aligned} & \text { In a } \\ & \text { mission } \\ & \text { context }\end{aligned}$

However, an aircraft specification is not restricted to performance requirements alone. Several operational requirements must be met as well (Table 3 ). In the given example, a dedicated cargo aircraft is characterised; aircraft with other main purposes like e.g. an unmanned reconnaissance vehicle can possibly be categorised by other operational requirements.
Table 3: Operational requirements

\begin{tabular}{|l|}
\hline \multicolumn{1}{c|}{ Operational requirements } \\
\hline - Quick re-role capability (cargo transport - passenger \\
transport - medevac aircraft) \\
- Pressurised cargo compartment \\
- Rapid centre of gravity shift capability \\
- In flight refuelling capability \\
- Quick cargo handling roll-on roll-off capability \\
- Ground operations on unprepared strips and without \\
ground handling means \\
- Survivability (military applications)
\end{tabular}

Most of these operational requirements are not immediately reflected in the above formula systems and performance models. The proposed scaling approach therefore includes an extended requirement model, enabling automated expansion of the above operational requirements into technical solutions with quantifiable effects on mass and drag, as well as further technical boundary conditions. These requirements are thus made compatible with the FASTR core formula system, which mostly relies on the above mentioned formulas and equations. A specified in flight refuelling capability, e.g., will be translated into the integration of a specific subsystem, a refuelling probe, with defined individual mass and drag properties. Other requirements will lead to the introduction of several restrictions in the aircraft's overall configuration. With this, an extensive requirement model as a guide for the scaling has been defined.

\section{Aircraft model}

For conceptual design considerations, an aircraft can be described by a set of variables, following a model in a sufficient grade of detail. In the FASTR approach, some 250 variables are currently used, describing a certain aircraft setup in the first part in terms of geometric key figures, furthermore propulsion, aerodynamic, and mass properties.

Necessarily, this variable model is complemented in a second part by several methods (see below) for parameter value determination of the variables of the first part. In this way, e.g. a certain wing area can be methodically associated with a certain mass and certain aerodynamic characteristics, and a resize of the former automatically yields changes in both latter variables.

In this methods-part, the "rubberised" propulsion device is calculated according to a generic engine model [5]. The prediction of a longitudinal aerodynamic dataset, including trim losses, relies on handbook methods [6], [7], [8]. Mass determination also relies on handbook methods [9], [10]. Additionally, the methods-part includes an automated rule-making functionality around a freely definable design point, e.g. a referenced baseline aircraft as described in Section 4, enabling even better model accuracy in parameter variations closely around that well-defined reference. The methods-part is designed to be exchangeable for different types of aircraft.

For the developing and validation phase of the FASTR approach, a conventional cargo aircraft configuration with 
a high wing is modelled. Since this model is exchangeable, however, it does not basically restrict the proposed scaling algorithm described in the following.

\section{Automatic scaling algorithm}

The described aircraft model is sized in the scaling process, as a whole or in part, in order to meet certain specified requirements - e.g. by increasing the tank volume, thus enabling a specified better mission range.

At the beginning of this proposed automated scaling process, a number of ground rules for the scaling, e.g. parameter value minimum/maximum envelopes, a master mission profile (see below), key figures like wing loading to be kept constant, or scaling boundary conditions used by the optimisation module (see below) can be set individually. Furthermore, in another pre-processing step, the process continues with the mentioned automated expansion of operational requirements (Table 3) into boundary conditions to be reflected during the scaling.

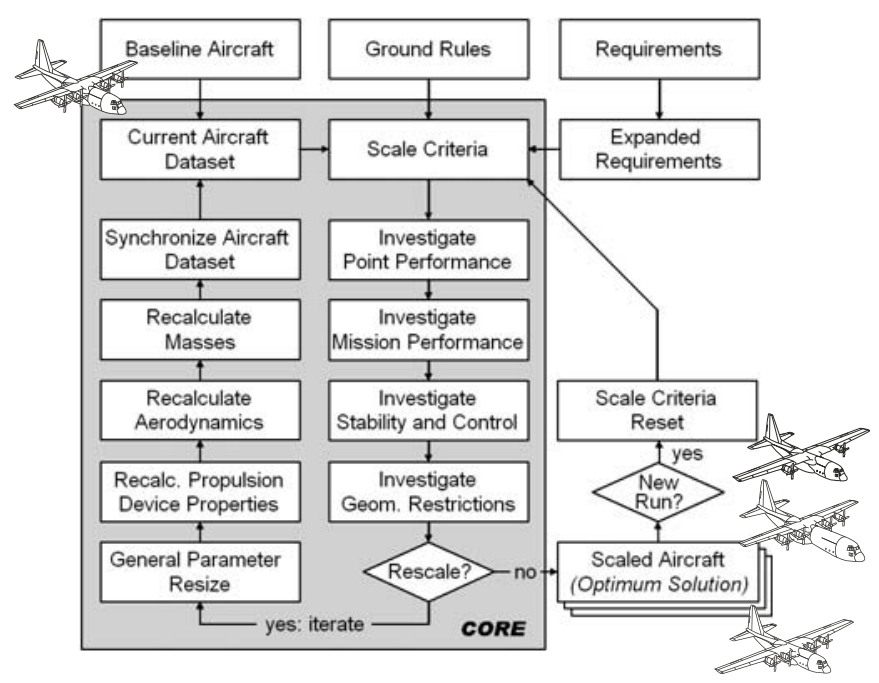

Fig. 2: Proposed scaling algorithm (simplified)

The sizing then starts from a certain baseline aircraft according to the aircraft model described in Section 3. This baseline design represents an in-itself consistent reference aircraft dataset and a metric for further investigation of scaling results. The baseline design, however, does not need to meet the required performance, and does not necessarily represent an optimum design with respect to any objective.

During the iterative scaling process, the current aircraft dataset is analysed in several modules in order to ascertain whether the given requirements can be satisfied, whether the current aircraft is over-designed, or whether it still lacks potential (Figure 2). This investigation focuses primarily on performance requirements. First, several required point performances listed in the aircraft specification are investigated (Table 1): here the performance figures of the current aircraft dataset are computed and checked against the requirements. If the current aircraft dataset over-qualifies or fails by a definable margin in this comparison, the responsible aircraft parameters are correspondingly marked for change.

In the next module, required mission performances are investigated as well (Table 2). The FASTR approach allows the flexible definition of a master mission profile with a modular construction system. Single mission segments, each defined separately, can be combined without any restriction, generating a mission which the current aircraft dataset "flies along" in any desired time resolution (Figure 3).

Moreover, a stability and control module is run to ensure certain aircraft handling qualities according to the requirements.

Finally, the compliance of the overall design with geometric restrictions, e.g. to prevent the blades of an upscaled propeller from touching the ground, is tested in a separate module.

At the end of this downloop a rescale decision is met: if the current aircraft design satisfies all required criteria investigated earlier, the algorithm terminates with a "tailor-made" solution to fit the specification. If, on the other hand, there is still a need for scaling, and the parameters in question are still within their value envelopes, a parameter resize is initiated.

A loop-back then allows scaling of the current aircraft design in terms of engine size, wing, empennage, and fuselage dimensions as well as fuel mass corresponding to tank volume. Currently, about 25 variables used in the aircraft model are subject to direct manipulation - e.g. wing area. The ground rules contain an initially definable list of parameters subject to change during the scaling, so that scaling investigations under specific restrictions, e.g. a resize of wing properties only, are possible. Along the design loop, associated changes in the other variables describing the propulsion device, aerodynamics and masses, which are indirectly triggered by that direct rescaling, are determined according to the methods-part of the aircraft model (Section 3).

This iterative process automatically resizes the baseline design towards the "tailor-made" design, aiming at the favoured minimum-mass solution. To decrease the run-time, the rescale algorithm relies on a Newton-solver, enabling quick detection of the wanted solution in only a few iterations. This scaled design is represented by a list of aircraft properties according to the used aircraft model, regarding geometry, propulsion, aerodynamics with drag as the dominating parameter, and mass. Calculated point performance data, mission performance results, and stability/control properties complete the representation.

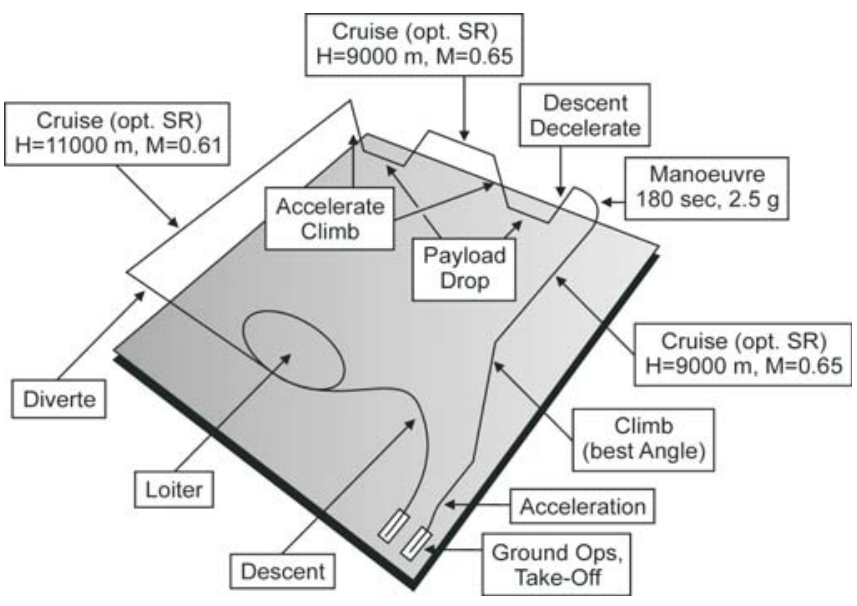

Fig. 3: Flexible mission model (supply mission example) 
A simple optimisation module finally evaluates the resulting scaled design regarding a selectable figure of merit, e.g. aircraft total mass, and decides on a new scaling run with slightly changed scale criteria. Thereby, several technical variants for solving same problem are investigated - e.g. a required high climb rate, which could be achieved with the help of either a huge wing, or a powerful propulsion device, or a sophisticated high-lift system, or a combination of all of these - and the best solution concerning this figure of merit is isolated.

At present, both aircraft model and requirement model refer to a dedicated cargo aircraft. The presented scaling methodology, however, is not restricted to this aircraft type. The same methodology is currently being used in a joint Academia-Industry research project for a scaling tool applicable for UAV conceptual design and scaling [11].

Running on a modern standard personal computer, a FASTR run typically takes less than a minute. It should be noted, however, that the described scaling method does not necessarily converge. In this case, the parameter value of one or several requirements prevents the algorithm from reaching a realisable aircraft; relying on an iteration counter, the algorithm will nevertheless terminate. Since the scaling log file will in any case display the then futile efforts to achieve the objectives, the FASTR approach also functions as a quick test for the feasibility of the requirements against the background of the baseline design. So in any case the FASTR approach yields results, which will be discussed in the following.

\section{Results of the scaling process}

With a number of optimisation module-controlled scaling processes, various scaled designs are available - each matching the requirements, but differing along a parameter list according to the individual ground rule-setting. Every single solution can be plotted in various diagrams, e.g. in a common design diagram showing power loading versus wing loading (Figure 4). Since each plotted point represents a complete design, several trends are determined, e.g. as defined by the aircraft total mass as a rough figure of merit, and the single solutions are evaluated individually. As additional guidelines, various boundaries are mapped which result from individual

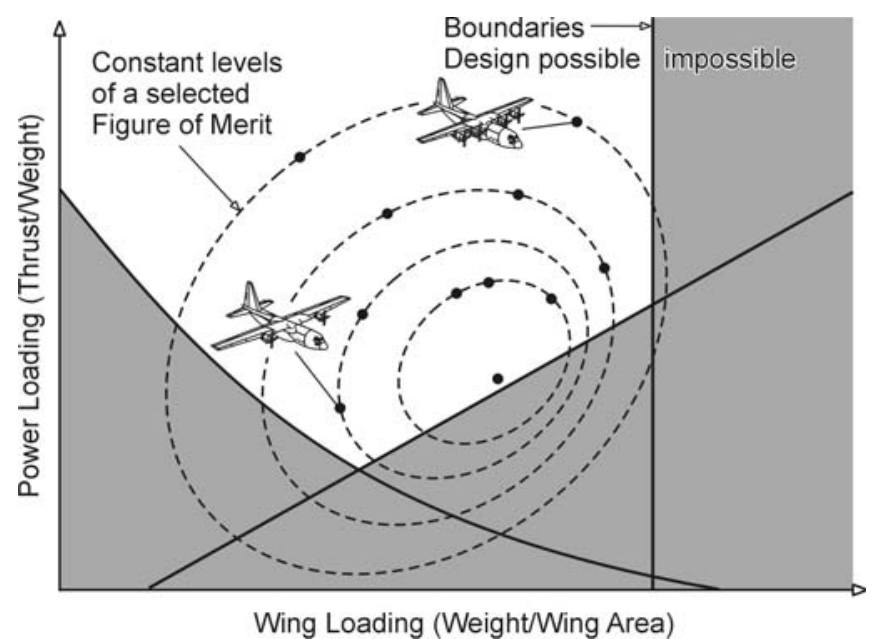

Fig. 4: Design diagram with plottings of evaluable solutions requirements: in the shaded areas those combinations of the variables are located which fail to satisfy certain requirements.

The data obtained implicates even more than a variety of solutions that can be visualized in the diagram below. For example, mass growth factors - i.e. values for the partial differentiations of the aircraft total mass relative to a certain required aircraft quality [12] - are available through dedicated FASTR runs. Thus the sensitivity of the baseline design concerning a certain requirement becomes apparent in terms of a total mass change. Specifically, this requirement may be any point performance requirement (see Table 1), or mission performance requirement (see Table 2), or additional operational requirement (see Table 3). Aircraft total mass as a figure of merit is currently used because of its implications, as there are methods available to easily derive rough cost and time schedule estimations from mass data [13]. Another parameter, e.g. aircraft total drag, could be investigated as well. With these results, the penalties - in terms of additional mass or drag - which have to be accepted in order to satisfy a certain requirement become clearly evident. So it is possible to make a critical review of the basic set of requirements, maybe slightly weakening the one or other desired parameter value, while focusing on a better overall performance in the end with respect to a definable figure of merit.

Moreover, as the FASTR algorithm with its optimisation module can be used to investigate several technical options for meeting the same requirement, a discussion of favoured basic technical approaches can be provided as well, including the minimum mass and minimum drag solutions.

Thus the resulting data adds to the available knowledge concerning the aircraft project in an early design phase. Moreover, it can be considered a valuable aid in the trade-off decision-making processes concerning design driving requirement parameter values or detail solution versus detail solution, as an example may demonstrate.

\section{Example}

In the example below, the search history of a scaling run is shown for selected key parameters (Figure 5). The automated scaling process resizes the mentioned cargo aircraft towards a certain required climb rate by changing engine static thrust, wing area, and lift coefficient respectively flap system, thus implicating a change in aircraft total mass. Interactions between lift coefficient and wing geometric properties are reflected in the calculations. Any of the vertically arranged parameter combinations in Figure 5 can be regarded as a possible solution, a result of a scaling core run (with the exception of iteration 0 , which represents the baseline design not yet fulfilling the improved requirement). It becomes apparent that the minimum-mass technical approach to improve climb rate values is to revise the high-lift system, thus allowing wing size and engine static thrust to shrink.

Relying on this result, the minimum mass solution for an e.g. $10 \%$ climb rate improvement and the corresponding mass penalties can be calculated (Table 4). Effects of a $10 \%$ climb rate reduction can be determined as well (Table 5). With this data the mass penalties or benefits of the realisation of a certain climb rate requirement are made obvious. Moreover, in this example the results indicate that the baseline aircraft features a certain design improvement potential concerning 
climb rate performance, since even a climb rate-reduced aircraft shows a possible mass reduction.

Note that the example diagram refers only to the mentioned climb rate performance improvements. Additional constraints, e.g. a shortened landing distance or a changed mission range performance, would yield different results.

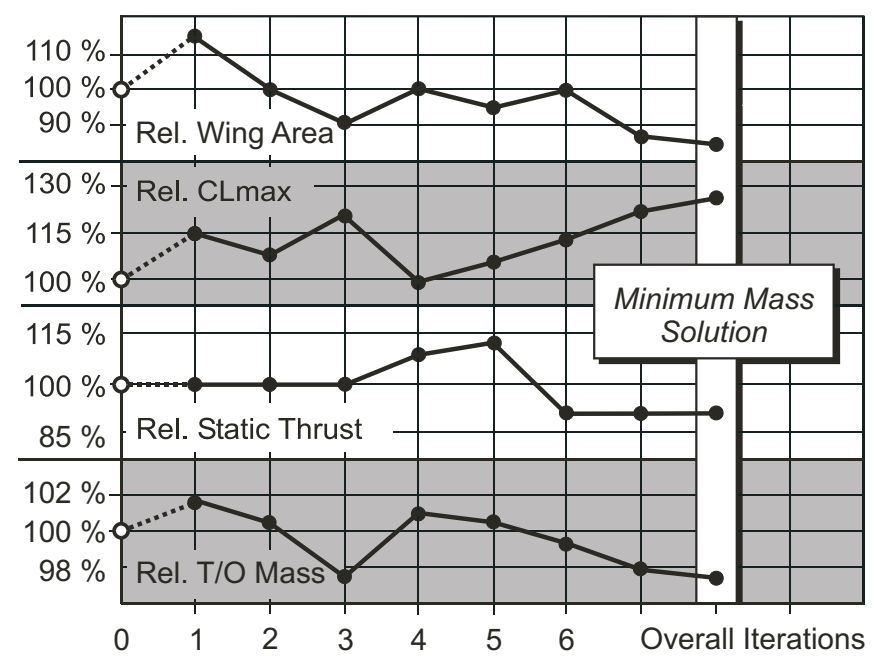

Fig. 5: Search history for climb rate improvement

Table 4: Climb rate improvement of $10 \%$

\begin{tabular}{|lr|}
\hline \multicolumn{2}{|c|}{$\begin{array}{c}\text { Climb rate improvement of 10 \% } \\
\text { Changes relative to Baseline }\end{array}$} \\
\hline Static thrust change & $-8.0 \%$ \\
Wing area change & $-17.0 \%$ \\
Max lift coefficient change & $+24.0 \%$ \\
Overall mass change & $-2.4 \%$ \\
\hline
\end{tabular}

Table 5: Climb rate reduction of $10 \%$

\begin{tabular}{|lr|}
\hline \multicolumn{2}{|c|}{ Climb rate reduction of $\mathbf{1 0} \%$} \\
Changes relative to Baseline \\
\hline Static thrust change & $-7.6 \%$ \\
Wing area change & $-15.4 \%$ \\
Max lift coefficient change & $0.0 \%$ \\
Overall mass change & $-3.3 \%$ \\
\hline
\end{tabular}

In addition to point performances, different technical approaches to satisfy a certain mission range requirement can be investigated as well. In Figure 6, the example baseline design is scaled in order to accommodate enough fuel for a given increased outbound range within a complex air supply mission profile (Figure 3): wing area and/or external fuel tank size are varied, and the overall changes in mass and zero drag are determined. It becomes obvious that a wing area increase is preferable to the installation of external fuel tanks, which would require pylons and additional piping and come along with increased zero drag, too.

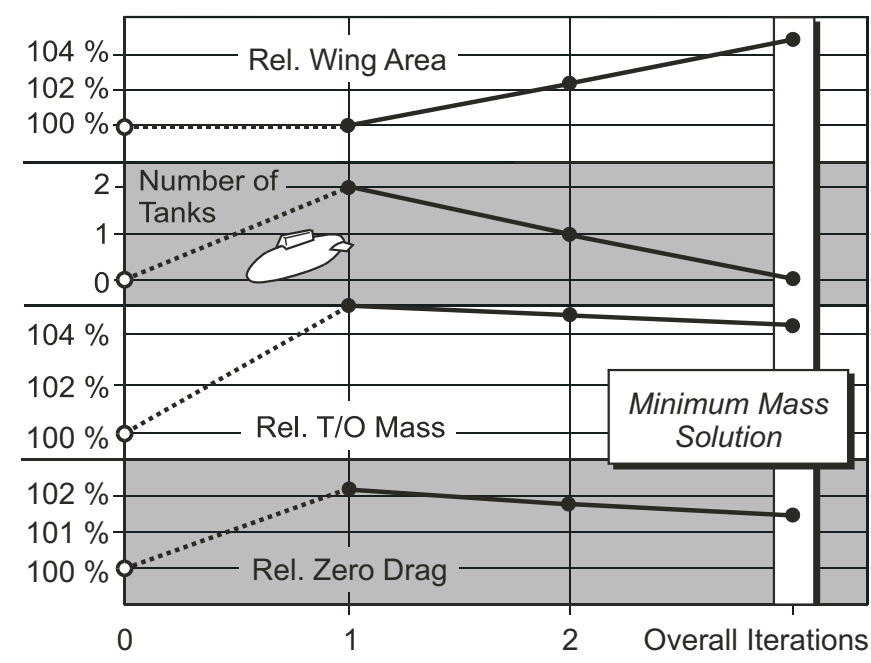

Fig. 6: Search history fors improved mission range

Relying on this recommended configuration, the mass and drag penalties for an outbound range increase of e.g. $10 \%$ or $20 \%$ can be investigated, and the required key basic parameters are provided by scaling runs (Table 6).

To finally include the above mentioned reflection of operational requirements (Table 3) in the example, the last column of Table 6 shows the penalties of a required in-flight refuelling capability, which was realised by integrating an aerial refuelling probe into the upper forehead of the cargo aircraft. For the current baseline aircraft, the probe integration is obviously acceptable, at least in terms of additional mass and drag.

These examples illustrate how trade-off decisions can be prepared substantially with the presented scaling methodology.

Table 6: Mission range calculation

\begin{tabular}{|l|c|c|c|}
\hline \multicolumn{3}{|c|}{ Mission range calculation } \\
Changes relative to Baseline \\
\hline & $\begin{array}{c}\text { Range } \\
+10 \%\end{array}$ & $\begin{array}{c}\text { Range } \\
+20 \%\end{array}$ & $\begin{array}{c}\text { Refueling } \\
\text { Probe }\end{array}$ \\
\hline$\Delta$ Fuel mass & $+4.6 \%$ & $+10.1 \%$ & $0.0 \%$ \\
$\Delta$ Structure mass & $+4.0 \%$ & $+6.4 \%$ & $+0.003 \%$ \\
$\Delta$ Overall mass & $+4.25 \%$ & $+7.9 \%$ & $+0.001 \%$ \\
$\Delta$ Wing area & $+4.3 \%$ & $+8.6 \%$ & $0.0 \%$ \\
$\Delta$ Fuselage length & $0.0 \%$ & $0.0 \%$ & $0.0 \%$ \\
$\Delta$ Wetted area & $+1.7 \%$ & $+3.4 \%$ & $+0.002 \%$ \\
$\Delta$ Zero drag & $+1.6 \%$ & $+3.3 \%$ & $+0.002 \%$ \\
\hline$\Delta$ Wing loading & $-0.05 \%$ & $-0.64 \%$ & $0.0 \%$ \\
$\Delta$ Thrust loading & $-4.08 \%$ & $-7.32 \%$ & $0.0 \%$ \\
\hline
\end{tabular}

\section{Conclusion}

A computer-based automatic scaling process, which is methodically not restricted to certain aircraft types, is described. An extended requirement model reflecting point, mission 
and operational performances has been introduced. An aircraft is represented in sufficient detail for conceptual design considerations by a set of variables and methods to enable the determination of their parameter values. An accordingly defined cargo aircraft model is then automatically resized with computer aid in the described FASTR approach, until it satisfies the extensive, yet freely definable set of requirements in an optimum solution with respect to a selectable figure of merit, e.g. overall mass or drag. Results available at the end of, as well as information gained along the scaling process, include growth factors and design sensitivities. Relying on this data, important trade-off decision-making processes during aircraft conceptual design are enabled and backed up with extended knowledge about the evolving aircraft.

\section{References}

[1] Mavris, D. N., DeLaurentis, D. A.: A Probabilistic Approach for examining Aircraft Feasibility and Viability. Aircraft Design 3/2000, Pergamon Press, Oxford, 2000

[2] Whitford, R.: The Benefits and Costs of Stealth from the Aircraft Designer's Viewpoint. Acta Polytechnica Vol. 40, January 2000, Prague

[3] Roskam, J.: Airplane Design, Part I-VII. DARcorporation, Kansas, 1988

[4] Torenbeek, E.: Synthesis of Subsonic Airplane Design. Delft University Press, Delft, 1982

[5] Wittmann, R.: Generisches Modell für propeller- und strahlbasierte Flugzeugantriebe. Internal Report LT-SA 01/6, Technische Universität München, Munich, 2001

[6] Schemensky, R. T.: Development of an Empirical Based Computer Program to Predict the Aerodynamic Characteristic of Aircraft. Technical Report AFFDL-TR-73-144, Volume 1, Wright-Patterson AFB, Ohio, 1973
[7] Polhamus, E. C.: Prediction of Vortex-Lift Characteristics Based on a Leading-Edge Suction Analogy. AIAA Paper 69-1133, Washington D.C., 1969

[8] N. N.: USAF Stability and Control DATCOM. Air Force Flight Dynamics Laboratory, Wright-Patterson AFB, Ohio, (Revised) 1978

[9] N. N.: Luftfahrttechnische Handbücher, LTH-Band Masseanalyse. IABG, Munich, February 1992

[10] N. N.: ESDU International Structures Series. ISSN 0141-4097, London, 2000

[11 Schieck, F., Deligiannidis, N., Gottmann, T.: A Flexible, Open-Structured Computer Based Approach for Aircraft Conceptual Design Optimisation. AIAA Paper 2002-0593, Washington D.C., 2002

[12] Ballhaus, W. F.: Clear Design Thinking using the Aircraft Growth Factor. Presentation, SAE Los Angeles Aeronautic Meeting, October 5-9, 1954

[13] Burns, J. W.: Aircraft Cost Estimation Methodology for Preliminary Design Development Applications. Presentation, SAWE Conference, 23-25 May 1994 (SAWE Paper No. 2228, Attachment E)

Dipl.-Ing. Florian Schieck

phone: +4989 28915986

e-mail: schieck@llt.mw.tum.de

Prof. Dr.-Ing. Dieter Schmitt

phone: +498928915981

e-mail: schmitt@llt.mw.tum.de

fax: +4989289 15982

Chair of Aeronautical Engineering

Technische Universität München

Boltzmannstraße 15, 85748 Garching, Germany 